\title{
A Revised Geometry of Mean-Variance Efficient Portfolios
}

\author{
Hans G. Ehrbar, Economics Department, University of Utah
}

Published in Metroeconomica 44(3):215-238, 1993

This version (with additional proofs) available at www.econ.utah.edu/ehrbar/research/rgep.pdf

\begin{abstract}
A revised definition of efficiency compares portfolios with different net worths. One axiom rules out instantaneous riskless gains, the other asserts that it costs money to buy good portfolios. Unlimited short sales are allowed. Portfolios which cannot be improved at any price are called absolutely efficient. The geometry of the meanstandard deviation frontiers is governed by three "efficient set constants." A few results regarding efficient portfolios must be modified, and a new version of the security line equation is proposed. Explicit formulas are given; no need to use different formulas for singular or nonsingular covariance matrix.
\end{abstract}

Hans G. Ehrbar

Economics Department, University of Utah

1645 E. Central Campus Dr. Front

Salt Lake City, UT 84112-9300

Direct office phone: (801) 5817797

Phone of Department office: (801) 5817481

Fax: (801) 5855649

E-mail: ehrbar@econ.utah.edu 


\section{A Revised Geometry of Mean-Variance Efficient Portfolios}

\section{Introduction}

In the ongoing controversy around the justification of mean-variance portfolio theory [Markowitz(1987), pp. 52-68], an axiomatic approach might offer new insights. In the present treatment, unlimited short sales are allowed, i.e. portfolios form a vector space. Two simple axioms are imposed postulating that there are no opportunities for instantaneous riskless gains, and that in at least one case more money buys better portfolios. The definition of mean-variance dominance is revised in order to meet the criticism of [Ehrbar(1990)]: a portfolio is not considered efficient if some other portfolio with lower net worth yields identical or better expected return and standard deviation.

This axiomatric approach yields the most general mathematical conditions under which mean-variance portfolio theory holds. On the other hand, by going back to first principles, the logical flaws in the usual formulation of this theory pointed out in [Ehrbar(1990)] can be remedied.

Section 2 presents the axioms and formulates them as algebraic conditions satisfied by the expected returns, variances, and net worths of the assets considered.

As a prelude to the central question, how the expected return and standard deviation of a portfolio is reflected in its price, Section 3 delineates the range of available $(\mu, \sigma)$ combinations. Here we encounter portfolios which minimize $\sigma$ for a given positive $\mu$, regardless of net worth. They are shown to be efficient; they provide a natural generalization of the riskless asset - which is different from the well-known "global" minimum variance portfolio (which is not efficient under the revised definition).

While Section 3 discusses an important subset of all efficient portfolios, Section 4 investigates its smallest linear superset, namely, the portfolios minimizing $\sigma$ subject to a given $\mu$ and net worth $w$. These portfolios have been characterized by Merton [Merton(1972)] and Roll [Roll(1977)], for the case of nonsingular covariance matrix, with the help of three "efficient set constants." We propose three alternative 
parameters, which remain finite even in the presence of a riskless asset. In this way, Merton and Roll's elegant method becomes a general parametrization of all possible efficient sets. In the present treatment, there is no need to use a special set of formulas if the covariance matrix is singular.

Among the minimum variance portfolios defined in Section 4, Section 5 selects those which are efficient. It also derives various implications for which the revised definition of efficiency makes a difference: It is no longer true that all portfolios on the rising branch of the frontier are efficient, but now it is true that the net worth of every efficient portfolio is uniquely determined by its expected return and standard deviation, and now it is true that a portfolio uncorrelated with an efficient portfolio always has a nonnegative expected payoff.

For those portfolios which are not efficient, Section 6 proposes and justifies a new extension of the market line equation to the case of no riskless asset which fits seamlessly into the framework developed here and, it will be argued, better preserves the properties which the market line is famous for if a riskless asset exists.

Section 7 establishes the link between the revised concept of efficiency and the portfolio choice problem of an investor with meanstandard deviation utility function, and Section 8 gives once more a brief summary.

The basic non-trivial proofs are given in the Appendix. An enlarged version of the paper with complete proofs is available from the author.

If you are reading this small-printed sentence here, you are holding the enlarged version in your hands. Everything not contained in the original paper is given in small print.

\section{Basic Definitions and Assumptions}

We will consider $n$ different types of capital assets or "securities" over one time period. The $n \times 1$ column vector $\boldsymbol{w}=\left[w_{1}, \ldots, w_{n}\right]^{\prime}$ gives the market values of "one" security of each type at the beginning of the period. Usually, but not necessarily, these securities are measured in such units that $\boldsymbol{w}$ is the vector $[1, \ldots, 1]^{\prime}$, i.e. every security starts out with market value 1 at the beginning of the period. At the end of the period, the securities will be liquidated, and their cash flow forms 
a random vector with mean $\boldsymbol{\mu}=\left[\mu_{1}, \ldots, \mu_{n}\right]^{\prime}$, the vector of "expected payoffs," and with a $n \times n$ covariance matrix $\Omega$ (which may be singular). Note that $\mu_{i}$ is principal plus interest yielded by "one" security of the $i$ th type. A portfolio $\boldsymbol{p}$ is defined by its investment vector $\boldsymbol{x}_{(p)}=\left[x_{1(p)}, \ldots, x_{n(p)}\right]^{\prime}$, indicating that $\boldsymbol{p}$ consists of $x_{i(p)}$ securities of type $i$. The $x_{i(p)}$ may be negative, i.e. short sales are allowed. The set of all portfolios forms, therefore, a $n$-dimensional vector space. The net worth of portfolio $\boldsymbol{p}$ will be denoted $w_{(p)}=\boldsymbol{w}^{\prime} \boldsymbol{x}_{(p)}$, its expected payoff is $\mu_{(p)}=\boldsymbol{\mu}^{\prime} \boldsymbol{x}_{(p)}$, and the standard deviation of its payoff is $\sigma_{(p)}=\sqrt{\boldsymbol{x}_{(p)}^{\prime} \boldsymbol{\Omega x}_{(p)}}$. If $w_{(p)} \neq 0$, the rate of return in its usual definition is $r_{(p)}=\left(\mu_{(p)}-w_{(p)}\right) / w_{(p)}$, and $\mu_{(p)} / w_{(p)}=1+r_{(p)}$ will be called the "rate of payoff." Portfolios with zero expected return, standard deviation, and net worth will be called "zero" portfolios. The quotation marks around "zero" are retained as a reminder that their investment vectors are not necessarily the null vector.

While usually the constraint $w_{(p)}=1$ is imposed, we allow $w_{(p)}$ to vary. Our definition of dominance, indicating which portfolios are unambiguously better than others, must therefore take account of the net worth of the securities:

Definition : $\quad$ Portfolio $\boldsymbol{p}$ dominates portfolio $\boldsymbol{q}$ if $\mu_{(p)} \geq \mu_{(q)}$, $\sigma_{(p)} \leq \sigma_{(q)}$, and $w_{(p)} \leq w_{(q)}$, with at least one of these three inequalities being strict. $\boldsymbol{p}$ is called efficient if no portfolio dominates it.

The usual definition, of course, leaves out every reference to net worth, which is assumed equal to 1 . As a consequence it calls certain portfolios efficient, for which other portfolios with lower net worth can be found that have better $(\mu, \sigma)$ combinations. As pointed out in [Ehrbar(1990)], the portfolios on the arc $\boldsymbol{g} \boldsymbol{t}$ in Figure 2 should not be called efficient.

Throughout this paper, we will make the following two assumptions regarding $\boldsymbol{w}, \boldsymbol{\mu}$, and $\boldsymbol{\Omega}$. One should expect the market to satisfy them if investors care about mean and standard deviation:

ASSUMPtion [i]: Any riskless portfolio with nonnegative expected payoff has nonnegative net worth.

If such a portfolio had negative net worth, then instantaneous riskless gains would be possible at no cost: the purchase of such a 
portfolio would give a positive cash flow at the beginning of the period and a nonnegative cash flow at the end.

Assumption [ii]: There is at least one portfolio with positive net worth that is not dominated by portfolios with zero or negative net worth.

If this assumption were violated, the investor's budget constraint would be irrelevant.

These two assumptions can be translated into two simple algebraic conditions:

Proposition [1]: Assumptions $[i]$ and $[i i]$ hold if and only if a vector $\gamma$ exists with

$$
\left(\Omega+\mu \mu^{\prime}\right) \gamma=w
$$

and

$$
\mu^{\prime} \gamma>0
$$

The formulas and proofs below will also use a vector $\boldsymbol{\delta}$ satisfying a condition symmetric to (1), namely

$$
\left(\Omega+\mu \mu^{\prime}\right) \delta=\mu .
$$

It always exists, independently of assumptions $[i]$ or $[i i]$. If (1) and (2) hold, one can show that

$$
\begin{gathered}
1 \geq \boldsymbol{\mu}^{\prime} \boldsymbol{\delta}>0 \\
\boldsymbol{w}^{\prime} \boldsymbol{\delta}=\boldsymbol{\mu}^{\prime} \boldsymbol{\gamma}
\end{gathered}
$$

and that, if there are more than one solution of (1) or (3), they differ from each other only by investment vectors of "zero" portfolios.

Algebraic formulas for possible solutions $\boldsymbol{\gamma}$ and $\boldsymbol{\delta}$ are $\boldsymbol{\gamma}=\left(\boldsymbol{\Omega}+\boldsymbol{\mu} \boldsymbol{\mu}^{\prime}\right)^{-} \boldsymbol{w}$ and $\boldsymbol{\delta}=\left(\boldsymbol{\Omega}+\boldsymbol{\mu} \boldsymbol{\mu}^{\prime}\right)^{-} \boldsymbol{\mu}$, where $\left(\boldsymbol{\Omega}+\boldsymbol{\mu} \boldsymbol{\mu}^{\prime}\right)^{-}$is some g-inverse of $\boldsymbol{\Omega}+$ $\boldsymbol{\mu} \boldsymbol{\mu}^{\prime}$. A g-inverse of a matrix $\boldsymbol{A}$ is, by definition, any matrix, designated $\boldsymbol{A}^{-}$, which satisfies $\boldsymbol{A} \boldsymbol{A}^{-} \boldsymbol{A}=\boldsymbol{A}$. It always exists but is not necessarily unique $[\operatorname{Rao}(1973)$, p. 24].

\section{Absolutely Efficient Portfolios.}

Assumption $[i i]$ stipulates that certain $(\mu, \sigma)$-combinations can only be attained by portfolios with positive net worth. This does not mean 
that one can always obtain better $(\mu, \sigma)$-combinations by spending more money. Some portfolios are "absolutely efficient," i.e. they cannot be improved upon even by investors with an unlimited budget. Absolutely efficient portfolios, which are defined without reference to $\boldsymbol{w}$, will be shown to be efficient as well. From their properties, two of the three parameters can be derived which characterize the efficient set.

Definition : Portfolio $\boldsymbol{t}$ is called absolutely efficient if all portfolios $\boldsymbol{q}$, regardless of net worth, for which $\mu_{(q)} \geq \mu_{(t)}$ and $\sigma_{(q)} \leq$ $\sigma_{(t)}$, satisfy $\mu_{(q)}=\mu_{(t)}$ and $\sigma_{(q)}=\sigma_{(t)}$.

If a risk-free portfolio exists with positive expected cash flow, then there are no absolutely efficient portfolios, since one can always improve a given $(\mu, \sigma)$ combination by adding this risk-free portfolio. What risk-free portfolios and absolutely efficient portfolios have in common is that they minimize standard deviation subject to a given expected payoff. We will call such portfolios "absolute minimum variance portfolios." They should not be confused with the known "global minimum variance portfolios," which minimize standard deviation subject to a given net worth.

Proposition [2]: Define $\pi \geq 0$ by

$$
\pi^{2}=\frac{1}{\boldsymbol{\mu}^{\prime} \boldsymbol{\delta}}-1
$$

All portfolios $\boldsymbol{p}$ satisfy

$$
\sigma_{(p)}^{2} \geq \pi^{2} \mu_{(p)}^{2}
$$

and a portfolio is an absolute minimum variance portfolio if and only if (7) is an equality. The investment vectors of absolute minimum variance portfolios are scalar multiples of solutions of (3).

An explicit formula for these investment vectors is therefore easy to come by:

Proposition [3]: Absolute minimum variance portfolios exist for arbitrary values of $\mu$. If $\boldsymbol{p}$ is an absolute minimum variance portfolio with expected return $\mu_{(p)}$, its investment vector has the form

$$
\boldsymbol{x}_{(p)}=\mu_{(p)}\left(1+\pi^{2}\right) \boldsymbol{\delta}+\boldsymbol{z}_{(p)}
$$

where $\boldsymbol{\delta}$ is a fixed but arbitrary solution of (3), and $\boldsymbol{z}_{(p)}$ is the investment vector of a "zero" portfolio. 
(7), as an equality, can also be written $\sigma_{(p)}=\pi\left|\mu_{(p)}\right|$. In the mean-standard deviation plane, the "absolute frontier" consists therefore of the two rays through the origin with slopes $\pm 1 / \pi$ (which become the $\mu$-axis if $\pi=0) . \pi$ indicates the lowest attainable " $t$-ratio" of standard deviation divided by expected payoff. $1 / \pi$ has been called "performance" in [Sharpe(1966)]. Looking ahead at Figure 1 below, one can discern these two rays as the envelopes of all frontiers. Figure 2 has these rays drawn in, truncated at the tangency point with the usual "frontier" of $(\mu, \sigma)$-combinations of all portfolios with net worth unity.

Proposition [2] used assumptions $[i]$ and $[i i]$ only insofar they imply $\boldsymbol{\mu} \neq \boldsymbol{o}$. A fuller use of the two assumptions yields a second parameter characterizing the efficient set. All absolute minimum variance portfolios have the same positive rate of payoff $\rho$ or, using the more familiar notion of "rate of return," they all have the same rate of return $i>-100 \%$. This rate should be considered the generalization of the risk-free rate of return.

$$
\begin{gathered}
\text { Proposition [4]: Define } \rho=1+i>0 \text { by } \\
\rho=\frac{\boldsymbol{\mu}^{\prime} \boldsymbol{\delta}}{\boldsymbol{w}^{\prime} \boldsymbol{\delta}} .
\end{gathered}
$$

Every absolute minimum variance portfolio $\boldsymbol{t}$ satisfies

$$
\mu_{(t)}=\rho w_{(t)} .
$$

The relationship between absolute minimum variance portfolios and absolutely efficient portfolios is simple and familiar: the absolutely efficient portfolios are located on the positively sloped portion of the absolute frontier, unless this frontier is vertical.

Proposition [5]: If $\pi=0$, then there are no absolutely efficient portfolios. If $\pi>0$, the absolutely efficient portfolios are exactly the absolute minimum variance portfolios with nonnegative expected payoff or, equivalently, nonnegative net worth. All risk-free portfolios and all absolutely efficient portfolios are efficient.

We defined a portfolio $\boldsymbol{p}$ to be efficient if and only if any other portfolio $\boldsymbol{q}$ with $\mu_{(q)} \geq \mu_{(p)}, \sigma_{(q)} \leq \sigma_{(p)}$, and $w_{(q)} \leq w_{(p)}$ satisfies the equalities $\mu_{(q)}=\mu_{(p)}, \sigma_{(q)}=\sigma_{(p)}$, and $w_{(q)}=w_{(p)}$. Proposition [5] says that for absolutely efficient portfolios, these three equalities already follow from the first two inequalities. Their $(\mu, \sigma)$ combination "locks in" their net worth. 


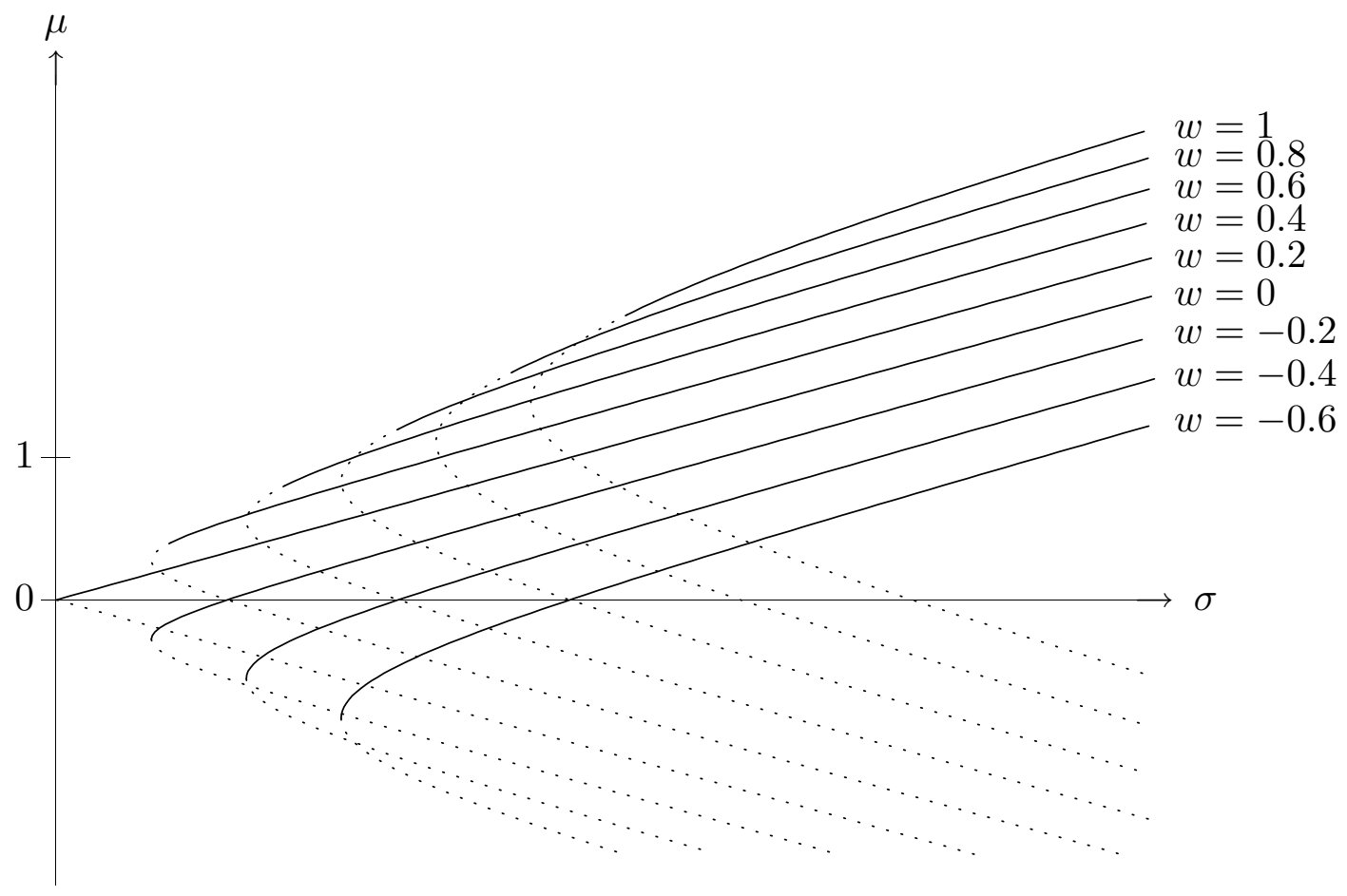

Figure 1. Frontiers for various values of $w$, dotted where they are not efficient

\section{Minimum Variance Portfolios}

In this section we will explore the set, in three-dimensional space, of all $(\mu, \sigma, w)$ combinations of "minimum variance" portfolios, i.e. of portfolios which minimize $\sigma$ for their given $\mu$ and $w$. This set is the surface area of an elliptical cone with vertex in the origin, which degenerates into a pair of halfplanes if $\tau \neq 0$ and $\pi=0$, and reduces to its two-dimensional counterpart, a pair of straight halflines emanating from the origin, if $\tau=0$. Its interest lies in its mathematical tractability and in the fact that the vector space of all minimum variance portfolios is spanned by the efficient portfolios. Figure 1 shows the intersections of a nondegenerate minimum variance surface with several planes $w=$ constant. These intersections, which we call frontiers, are shown as solid lines where the efficient portfolios are located and as dotted lines otherwise. Since Figure 1 may give a misleading perspectivic impression, Figure 2 shows what this surface (with the frontiers now as level lines) looks like if one cuts off everything 
located above the plane $w=1$, and looks at it from straight above. In other words, Figure 2 shows that part of the minimum variance surface accessible to an investor with total wealth $\bar{w}=1$, who selects his portfolios subject to an inequality wealth constraint. Figures 1 and 2 also differ in that Figure 2 follows the convention of labeling the axes in terms of rates of return instead of total payoffs.

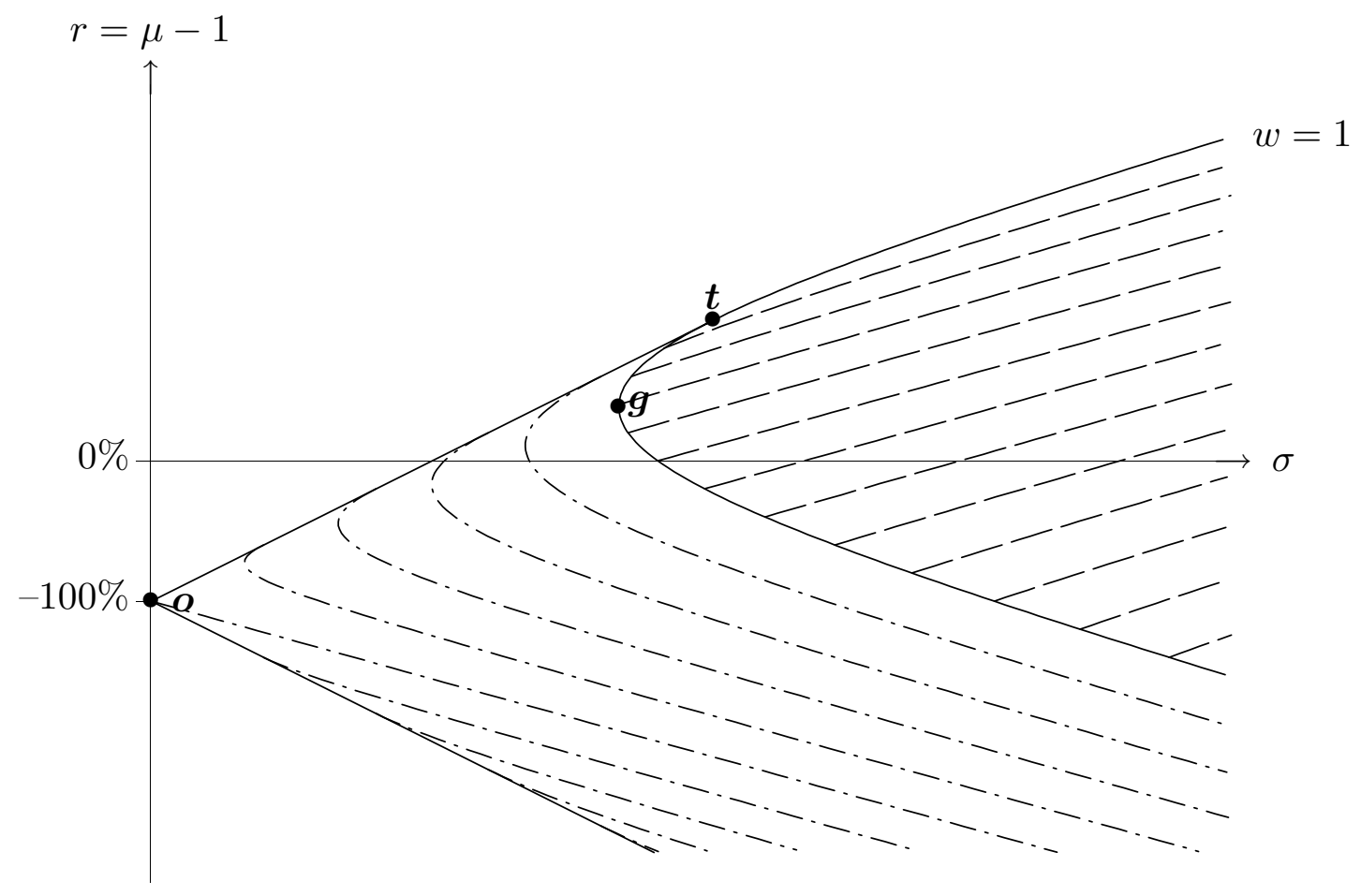

Figure 2. View from above of the minimum variance cone-surface, truncated at the plane $w=1$

The shape of the minimum variance set is governed by three "efficient set constants." Two of the constants, $\pi$ and $\rho$, have already been introduced. The third constant, $\tau$, indicates the rate at which excess variance can be traded for (squared) excess return, where "excess" means in excess of absolute minimum variance portfolios.

Proposition [6]: Take $\pi \geq 0$ and $\rho>0$ as defined in equa- 
tions (6) and (9), and define $\tau \geq 0$ by

$$
\tau^{2}=\boldsymbol{\mu}^{\prime} \boldsymbol{\delta}\left(\frac{\left(\boldsymbol{w}^{\prime} \boldsymbol{\gamma}\right)\left(\boldsymbol{\mu}^{\prime} \boldsymbol{\delta}\right)}{\left(\boldsymbol{w}^{\prime} \boldsymbol{\delta}\right)^{2}}-1\right)
$$

All portfolios $\boldsymbol{p}$ satisfy

$$
\tau^{2}\left(\sigma_{(p)}^{2}-\pi^{2} \mu_{(p)}^{2}\right) \geq\left(\mu_{(p)}-\rho w_{(p)}\right)^{2} .
$$

If $\tau>0$, then $\boldsymbol{p}$ is a minimum variance portfolio if and only if it satisfies (12) with equality. Its investment vector is a linear combination of solutions of (1) and (3). If $\tau=0$, then the minimum variance portfolios are exactly the absolute minimum variance portfolios.

Investment vectors have a nice, symmetric formula:

Proposition [7]: If $\tau>0$, minimum variance portfolios $\boldsymbol{p}$ exist for arbitrary values of $w_{(p)}$ and $\mu_{(p)}$. Their investment vectors can be written in the form

$$
\boldsymbol{x}_{(p)}=[\boldsymbol{\gamma} \mid \boldsymbol{\delta}]\left[\begin{array}{cc}
\rho^{2} / \tau^{2} & -\rho / \tau^{2} \\
-\rho / \tau^{2} & 1+\pi^{2}+1 / \tau^{2}
\end{array}\right]\left[\begin{array}{l}
w_{(p)} \\
\mu_{(p)}
\end{array}\right]+\boldsymbol{z}_{(p)}
$$

where $\boldsymbol{\gamma}$ and $\boldsymbol{\delta}$ are, as always, fixed but arbitrary solutions of (1) and (3), and the $\boldsymbol{z}_{(p)}$ are investment vectors of "zero" portfolios. If $\tau=0$, all portfolios have the same rate of payoff $\rho$, and the investment vectors of all minimum variance portfolios $\boldsymbol{t}$ with expected payoff $\mu_{(t)}$ (and therefore net worth $w_{(t)}=\mu_{(t)} / \rho$ ) have the form (8).

For $\tau>0$, (12) can be rearranged to give (14), which is [Buser(1977), equation $(8)]$ :

$$
\sigma_{(p)}^{2} \geq \frac{\pi^{2} \rho^{2}}{1+\pi^{2} \tau^{2}} w_{(p)}^{2}+\frac{1+\pi^{2} \tau^{2}}{\tau^{2}}\left(\mu_{(p)}-\frac{\rho}{1+\pi^{2} \tau^{2}} w_{(p)}\right)^{2} .
$$

From this one can see that the variance of the "global minimum variance portfolio" $\boldsymbol{g}$ with net worth 1 is $\pi^{2} \rho^{2} /\left(1+\pi^{2} \tau^{2}\right)$, and its expected payoff is $\rho /\left(1+\pi^{2} \tau^{2}\right)$. These formulas for $\boldsymbol{g}$ remain valid if $\tau=0$.

Here is summary of the properties of "absolute" and "global" minimum variance portfolios.

PROPOSITION $[i]: \quad \boldsymbol{t}$ is an absolute minimum variance portfolio iff $\sigma_{(t)}^{2}=$ $\pi^{2} \mu_{(t)}^{2}$ iff it is a minimum variance portfolio with $\mu_{(t)}=\rho w_{(t)} \cdot \boldsymbol{g}$ is a global minimum variance portfolio iff $\left(1+\pi^{2} \tau^{2}\right) \sigma_{(g)}^{2}=\pi^{2} \rho^{2} w_{(g)}^{2}$ iff it is a minimum variance portfolio with $\left(1+\pi^{2} \tau^{2}\right) \mu_{(g)}=\rho w_{(g)}$. 
In Figures 1 and 2, the efficient set constants have the values $\pi=2, \rho=2$, and $\tau=1 / 3$. Different values would give, besides a different scaling of the axes, also a different relative slope between the envelope and the asymptotes of the individual frontiers. If $\tau=0$, the minimum variance surface degenerates to a line, i.e. each frontier degenerates to a point. If a riskless asset exists, i.e. if $\pi=0$, then the envelope of all frontiers is the $\mu$-axis, and every frontier for a given net worth is a pair of rays emanating from this axis or, if in addition $\tau=0$, a point on this axis. The values of the efficient set constants do not disclose whether there are any portfolios which are not minimum variance portfolios. There are always two possibilities: either there are no other portfolios than the minimum variance ones, or for every value of $\sigma^{2}$ which is greater than the minimum, portfolios exist which have this variance.

For every triple of numbers $\pi \geq 0, \rho>0$, and $\tau \geq 0$ one can specify a vector space of assets for which these are the efficient set constants, by setting

$\boldsymbol{\mu}=\frac{1}{1+\pi^{2}}\left[\begin{array}{c}\frac{1}{\rho} \\ 1\end{array}\right], \quad \boldsymbol{w}=\left[\begin{array}{c}\frac{1}{\rho^{2}}\left(\tau^{2}+\frac{1}{1+\pi^{2}}\right) \\ \frac{1}{\rho\left(1+\pi^{2}\right)}\end{array}\right], \quad \boldsymbol{\Omega}=\left[\begin{array}{cc}\frac{1}{\rho^{2}}\left(\tau^{2}+\frac{\pi^{2}}{\left(1+\pi^{2}\right)^{2}}\right) & \frac{\pi^{2}}{\rho\left(1+\pi^{2}\right)^{2}} \\ \frac{\pi^{2}}{\rho\left(1+\pi^{2}\right)^{2}} & \frac{\pi^{2}}{\left(1+\pi^{2}\right)^{2}}\end{array}\right]$.

Since in this case $\gamma=\left[\begin{array}{l}1 \\ 0\end{array}\right]$ and $\boldsymbol{\delta}=\left[\begin{array}{l}0 \\ 1\end{array}\right]$, this example provides at the same time formulas for the expected returns, net worths, variances, and covariances of $\boldsymbol{\gamma}$ and $\boldsymbol{\delta}$.

Merton [Merton(1972)] and Roll [Roll(1977)] use the elements of $\left[\begin{array}{ll}\boldsymbol{w}^{\prime} \boldsymbol{\Omega}^{-1} \boldsymbol{w} & \boldsymbol{w}^{\prime} \boldsymbol{\Omega}^{-1} \boldsymbol{\mu} \\ \boldsymbol{\mu}^{\prime} \boldsymbol{\Omega}^{-1} \boldsymbol{w} & \boldsymbol{\mu}^{\prime} \boldsymbol{\Omega}^{-1} \boldsymbol{\mu}\end{array}\right]$, which are called $\left[\begin{array}{cc}c & b \\ b & a\end{array}\right]$ in Roll's notation, as their efficient set constants for nonsingular $\boldsymbol{\Omega}$. From nonsingularity follows $[\operatorname{Rao}(1973)$, p. 33]

$$
\left(\boldsymbol{\Omega}+\boldsymbol{\mu} \boldsymbol{\mu}^{\prime}\right)^{-1}=\boldsymbol{\Omega}^{-1}-\frac{\boldsymbol{\Omega}^{-1} \boldsymbol{\mu} \boldsymbol{\mu}^{\prime} \boldsymbol{\Omega}^{-1}}{1+\boldsymbol{\mu}^{\prime} \boldsymbol{\Omega}^{-1} \boldsymbol{\mu}}
$$

therefore $\gamma=\left(\boldsymbol{\Omega}+\boldsymbol{\mu} \boldsymbol{\mu}^{\prime}\right)^{-1} \boldsymbol{w}$ and $\boldsymbol{\delta}=\left(\boldsymbol{\Omega}+\boldsymbol{\mu} \boldsymbol{\mu}^{\prime}\right)^{-1} \boldsymbol{\mu}$ are uniquely defined. Using (15) together with (6), (9), and (11), one obtains $\pi^{2}=1 / a, \rho=a / b$, and $\tau^{2}=a\left(a c-b^{2}\right) / b^{2}$. Inversely, $a=1 / \pi^{2}$, $b=1 /\left(\pi^{2} \rho\right)$, and $c=\left(1+\pi^{2} \tau^{2}\right) /\left(\pi^{2} \rho^{2}\right)$. $a, b$, and $c$ become infinite as $\pi^{2} \rightarrow 0$.

\section{Which Minimum Variance Portfolios are Efficient?}

In terms of single frontiers, i.e. of intersections of the minimum variance set with planes $w=$ constant, the efficient portfolios can be 
characterized as follows. If $\tau=0$, every frontier consists of one point only, which represents an efficient portfolio. If $\tau>0$, one has to distinguish whether the portfolio has negative net worth or not. A portfolio with nonnegative net worth is efficient if and only if it is located on the portion of the frontier above and including the portfolio $\boldsymbol{t}$, whose tangent intersects the $\mu$-axis in the origin. A portfolio with negative net worth is efficient if and only if it is located on the rising portion of the frontier (starting from and including the global minimum variance portfolio $\boldsymbol{g}$ ). This is equivalent to the following algebraic condition:

The efficient portfolios must be on the surface looking north (maximize $\mu$ ), looking down (minimize $w$ ), and looking west (minimizing $\sigma$ ). Looking north and west means: the level lines must have positive slope, i.e. go from southwest to northeast. Looking down means: level lines for higher $w$ must be above, i.e. northwest of, those for lower $w$. For $w>0$, the looking-down condition is satisfied only for those portfolios on the part of the frontier above its tangency with the envelope of all frontiers. For $w \leq 0$, all portfolios on the rising branch of the fronier are efficient. Efficient portfolios are indicated by solid lines in Figure 1.

Proposition [8]: Portfolio $\boldsymbol{p}$ is efficient if and only if it is a minimum variance portfolio which satisfies both

$$
\begin{aligned}
\mu_{(p)} & \geq \rho w_{(p)} \quad \text { and } \\
\left(1+\pi^{2} \tau^{2}\right) \mu_{(p)} & \geq \rho w_{(p)} .
\end{aligned}
$$

The most obvious modification which the present revision brings to the usual mean-variance theory is therefore that, in the case of positive net worth, some portfolios on the rising branch of the frontier are not efficient. There are other changes. Let us look at the range of values for which efficient portfolios exist:

Proposition [9]: If $\tau=0$, efficient portfolios exist for every $\mu$, and their standard deviation is $\sigma=\pi|\mu|$. If $\tau \neq 0$, efficient portfolios exist vor every $\mu$ and $\sigma \geq 0$ satisfying

$$
-\frac{\sigma}{\sqrt{1+\pi^{2} \tau^{2}}} \leq \pi \mu \leq \sigma .
$$

For every efficient portfolio, the net worth is uniquely determined by its $\mu$ and $\sigma$ :

$$
w=\frac{\mu}{\rho}-\frac{\tau}{\rho} \sqrt{\sigma^{2}-\pi^{2} \mu^{2}}
$$


Although it is little advertised, the usual definition does not exclude the possibility that two efficient portfolios exist with same $\mu$ and $\sigma$ but different net worths. While one of them has the minimum net worth for the given $(\mu, \sigma)$ combination, the other one has the maximum net worth: for it, the wealth constraint is binding from below.

Also the following two propositions hold under the revised definition of efficiency, but not under the usual one:

Proposition [10]: If $\boldsymbol{p}$ and $\boldsymbol{q}$ are efficient portfolios with nonnegative net worths, then their covariance has the lower bound

$$
\operatorname{cov}(\boldsymbol{q}, \boldsymbol{p}) \geq \pi^{2} \rho^{2} w_{(q)} w_{(p)} .
$$

Equality holds if and only if both portfolios are absolutely efficient.

If $\boldsymbol{p}$ and $\boldsymbol{q}$ were allowed to range over all portfolios on the rising branch of the boundary, one would have to relax this inequality to $\operatorname{cov}(\boldsymbol{p}, \boldsymbol{q}) \geq \pi^{2} \rho^{2} w_{(p)} w_{(q)} /\left(1+\pi^{2} \tau^{2}\right)$.

In this case, equality would hold if both portfolios were "global" minimum variance portfolios.

Proposition [11]: Let $\boldsymbol{p}$ be an efficient portfolio with $\sigma_{(p)}>$ 0 , and $\boldsymbol{q}$ an arbitrary, not necessarily efficient portfolio. In the special case that $\boldsymbol{p}$ is a "global" minimum variance portfolio with $w_{(p)}<0$, $\operatorname{cov}(\boldsymbol{q}, \boldsymbol{p})$ is positive (negative, zero) if and only if $w_{(q)}$ is negative (positive, zero). Otherwise, $\operatorname{cov}(\boldsymbol{q}, \boldsymbol{p})$ is positive (negative, zero) if and only if $\mu_{(q)}-\theta_{(p)} w_{(q)}$ is positive (negative, zero), where

$$
\theta_{(p)}= \begin{cases}0 & \begin{array}{ll}
\text { if } \boldsymbol{p} \text { is an absolute minimum } \\
\text { variance portfolio }
\end{array} \\
\rho \frac{\mu_{(p)}-\rho w_{(p)}}{\left(1+\pi^{2} \tau^{2}\right) \mu_{(p)}-\rho w_{(p)}} \geq 0 & \text { otherwise. }\end{cases}
$$

$\theta_{(p)}$ is the height at which the tangent to the frontier in $\boldsymbol{p}$ intersects the $\mu$-axis, divided by $\mu_{(p)}$.

Under the usual definition of efficiency, $\theta_{(p)} \geq 0$ does not generally hold. Certain portfolios are therefore called efficient, for which negatively related portfolios exist with negative net worth and positive expected return. Adding a little bit of such a portfolio decreases the variance, decreases the net worth, and increases the expected return of the so-called efficient portfolio. 


\section{The Market Line Equation}

The net worth of an efficient portfolio is determined by its expected payoff and standard deviation. What can be said about portfolios which are not efficient? In the presence of a riskless asset, their payoff in excess of that of an equal investment in the riskless asset, and therefore their net worth, can be explained by the contribution which they make to the variance of any minimum variance "reference portfolio" $\boldsymbol{p}$ of which they are a part, which is usually taken to be the market portfolio. The "market line equation" reads

$$
\mu_{(q)}-\rho w_{(q)}=\beta_{(q \cdot p)}\left(\mu_{(p)}-\rho w_{(p)}\right) \quad \text { where } \quad \beta_{(q \cdot p)}=\frac{\operatorname{cov}(\boldsymbol{q}, \boldsymbol{p})}{\operatorname{var}(\boldsymbol{p})} .
$$

Besides $\sigma_{(p)}>0$ it assumes $\pi=0 ; \rho$ denotes the risk-free rate of return.

The usual generalization of (22) to the case without riskless portfolios replaces $\rho$ by the $\theta_{(p)}$ defined in $(21)$, i.e. it replaces the excess payoff over the riskless portfolio by that over portfolios uncorrelated with $\boldsymbol{p}$. The disadvantage of this generalization is that the point of reference for the "excess payoff" varies as $\boldsymbol{p}$ varies.

Here is a formulation of this usual generalization: Whenever $\boldsymbol{p}$ is a minimum variance portfolio but not a global minimum variance portfolio, the following relationship holds for any $\boldsymbol{q}$ :

$$
\mu_{(q)}-\theta_{(p)} w_{(q)}=\beta_{(q \cdot p)}\left(\mu_{(p)}-\theta_{(p)} w_{(p)}\right) \quad \text { where } \quad \beta_{(q \cdot p)}=\frac{\operatorname{cov}(\boldsymbol{q}, \boldsymbol{p})}{\operatorname{var}(\boldsymbol{p})} .
$$

We propose here an alternative generalization of (22), which uses the rate of payoff of absolutely efficient portfolios if there is no riskless rate of payoff. A revision of the definition of the "beta" gives it general validity:

$\mu_{(q)}-\rho w_{(q)}=\beta_{(q \cdot p)}^{(r)}\left(\mu_{(p)}-\rho w_{(p)}\right) \quad$ where $\quad \beta_{(q \cdot p)}^{(r)}=\frac{\operatorname{cov}(\boldsymbol{q}, \boldsymbol{p})-\pi^{2} \mu_{(q)} \mu_{(p)}}{\operatorname{var}(\boldsymbol{p})-\pi^{2} \mu_{(p)}^{2}}$.

(23) holds for arbitrary portfolios $\boldsymbol{q}$ and any minimum variance portfolio $\boldsymbol{p}$ which is not an absolute minimum variance portfolio.

Here is a justification of the proposed new definition of the "beta." The original $\beta_{(q . p)}$ in (22) has its name because it is the (true) regression coefficient of $\boldsymbol{q}$ on $\boldsymbol{p}$, i.e. because $\mu_{(q)}+\beta_{(q . p)}\left(\boldsymbol{p}-\mu_{(p)}\right)$ is the 
best linear predictor of $\boldsymbol{q}$ in terms of $\boldsymbol{p}$. As long as there is a riskless asset - say $\boldsymbol{u}$ yields a payoff unity with certainty - one can construct a portfolio which makes this prediction: $\boldsymbol{q}^{*}=\mu_{(q)} \boldsymbol{u}+\beta_{(q \cdot p)}\left(\boldsymbol{p}-\mu_{(p)} \boldsymbol{u}\right)$ is that portfolio using only $\boldsymbol{p}$ and $\boldsymbol{u}$. In other words, it is that minimum variance portfolio which is most similar to $\boldsymbol{q}$. It is unique up to a zero portfolio. The criterion for similarity is the mean squared error $\mathcal{E}\left[\left(\boldsymbol{q}^{*}-\boldsymbol{q}\right)^{2}\right]$.

The definition of the revised $\beta_{(q . p)}^{(r)}$ in (23) uses the construction of a most similar minimum variance portfolio as its guiding principle. If there are no riskless portfolios, define $\boldsymbol{u}$ to be the absolutely efficient portfolio with expected payoff $\mu_{(u)}=1$. Then the linear combination of $\boldsymbol{p}$ and $\boldsymbol{u}$ which is most similar to $\boldsymbol{q}$ is

$$
\boldsymbol{q}^{*}=\mu_{(q)} \boldsymbol{u}+\beta_{(q \cdot p)}^{(r)}\left(\boldsymbol{p}-\mu_{(p)} \boldsymbol{u}\right)
$$

While (22) relates excess payoffs to variances and covariances, it is plausible that, in the absence of a riskless asset, excess payoffs must be related to excess variances and "excess covariances," as done in (23). As in the riskless case [Fama(1976), pp. 59/60], the "excess covariance" can be viewed as the contribution of $\boldsymbol{q}$ to the excess variance of $\boldsymbol{p}$ : If portfolio $\boldsymbol{p}=\boldsymbol{q}+\boldsymbol{r}$, then the excess variance of $\boldsymbol{p}$ can be written

$\operatorname{var}(\boldsymbol{p})-\pi^{2} \mu_{(p)}^{2}=\left(\operatorname{cov}(\boldsymbol{q}, \boldsymbol{p})-\pi^{2} \mu_{(q)} \mu_{(p)}\right)+\left(\operatorname{cov}(\boldsymbol{r}, \boldsymbol{p})-\pi^{2} \mu_{(r)} \mu_{(p)}\right)$.

In other words, (23) carries all desirable features of (22) over to the case without a riskless asset - and as a byproduct confirms that absolutely efficient portfolios are the natural generalization of the riskless asset.

\section{Inequality Constrained Utility Maximization}

Efficient portfolios are exactly the portfolios chosen by investors who maximize a mean-standard deviation utility function $u\left(\boldsymbol{\mu}^{\prime} \boldsymbol{x}, \sqrt{\boldsymbol{x}^{\prime} \boldsymbol{\Omega} \boldsymbol{x}}\right)$ subject to an inequality budget constraint $\boldsymbol{w}^{\prime} \boldsymbol{x} \leq \bar{w}$, and who want to spend as little money as possible for their desired $(\mu, \sigma)$ combination. All mean-standard deviation utility functions are assumed to be strictly increasing in mean and strictly decreasing in standard deviation. 
Due to the additional requirement of not spending more than necessary, not every efficient portfolio that satisfies the budget constraint will be picked by such an investor. He restricts his search to a subset of those, namely, such portfolios $\boldsymbol{p}$ within his budget set whose $(\mu, \sigma)$ combination cannot be improved by any other portfolio in his budget set. We will call this the investor's choice set. It contains all efficient portfolios $\boldsymbol{p}$ with net worth $w_{(p)}=\bar{w}$, and in addition all absolutely efficient portfolios $\boldsymbol{t}$ with net worth $w_{(t)}<\bar{w}$. This is the whole choice set.

If $\bar{w}=1$, the investor's choice set consists of all portfolios located on the straight line segment from the origin $\boldsymbol{o}$ to $\boldsymbol{t}$ in Figure 2, plus those on the frontier for $w=1$ located above $\boldsymbol{t}$.

\section{Summary and Conclusions}

The treatment of mean-variance portfolio theory offered here has the following ingredients: (a) the assumption of unlimited short sales; (b) two very weak basic assumptions regarding the market, one saying that one cannot get money for free, and the other that money matters; and (c) the assumption that investors will not invest their full wealth if investing less gives them an equal or better $(\mu, \sigma)$ combination.

The first result derived from these ingredients is something which is easily overlooked if one works with investment proportions rather than the total amounts of investments: In a mean-variance world, less money can sometimes buy better portfolios than more money. Those portfolios whose $(\mu, \sigma)$ combination cannot be improved by portfolios with higher net worth, the "absolutely efficient" portfolios $\boldsymbol{t}$, take the central role in our discussion which the usual treatments of mean-variance portfolio theory award to the "global minimum variance portfolio" $\boldsymbol{g}$. This switch from $\boldsymbol{g}$ to $\boldsymbol{t}$ is necessary as long as one deals with portfolios with nonnegative net worth. $\boldsymbol{g}$ is not efficient if it has positive net worth, while absolute minimum variance portfolios are not efficient if they have negative net worth.

Strictly speaking, this is only true if there is a difference between $\boldsymbol{g}$ and $\boldsymbol{t}$.

It follows from the axioms that all absolutely efficient portfolios have the same rate of return, which is higher than $-100 \%$, and which provides a market parameter that specializes to the risk-free rate of return when a risk-free portfolio exists. This rate of return, together 
with two other market constants, characterize the geometry of all efficient combinations of net worth, expected payoff, and standard deviation. We used a mathematical apparatus tailored to the two axioms; its main difference to the usual procedures is that it uses inverses of $\boldsymbol{\Omega}+\boldsymbol{\mu} \boldsymbol{\mu}^{\prime}$ instead of inverses of $\boldsymbol{\Omega}$.

In this formulation, a generalized inverse is considered an inverse.

Instead of adding more complications, as one might think at first, the revised definition endowes efficient portfolios with intuitive properties, some of which are occasionally (and incorrectly) believed to be true already under the old definition. Regarding portfolios which are not efficient, a new formulation of the market line equation works with a more satisfactory definition of excess payoffs, which it relates to excess variances and covariances, rather than the variances and covariances themselves. "Excess" means here always: in excess of the risk-free or absolutely efficient portfolios.

Following the complementary slackness principle, the choice set of an investor with mean-variance utility function splits into two components: all efficient portfolios that satisfy the wealth constraint with equality, plus all absolutely efficient portfolios for which the wealth constraint is not binding.

The above ingredients not only lead to a logically more compelling theory, but also allow for a mathematical apparatus which, once the shortest proofs are found, promises to be a little less "tortuous" and "involved" [Fama(1976), p. 279].

\section{APPENDIX}

Proof that (3) always has a solution, expressible by the solution formula given after equation (5): To show that

$$
\boldsymbol{\mu}=\left(\boldsymbol{\Omega}+\boldsymbol{\mu} \boldsymbol{\mu}^{\prime}\right)\left(\boldsymbol{\Omega}+\boldsymbol{\mu} \boldsymbol{\mu}^{\prime}\right)^{-} \boldsymbol{\mu},
$$

find an $\boldsymbol{R}$ with $\boldsymbol{\Omega}=\boldsymbol{R} \boldsymbol{R}^{\prime}$ and apply the identity

$$
\boldsymbol{A}-\boldsymbol{A} \boldsymbol{A}^{\prime}\left(\boldsymbol{A} \boldsymbol{A}^{\prime}\right)^{-} \boldsymbol{A}=\boldsymbol{O}
$$

to $\boldsymbol{A}=\left[\begin{array}{ll}\boldsymbol{R} & \boldsymbol{\mu}\end{array}\right]$. To show (27), postmultipy the lefthand side by its transpose and check that the product is $\boldsymbol{O}$. Source: $[\operatorname{Rao}(1973)$, (1b.5.5) on p. 26]. 
The proof that $\boldsymbol{\gamma}=\left(\boldsymbol{\Omega}+\boldsymbol{\mu} \boldsymbol{\mu}^{\prime}\right)^{-} \boldsymbol{w}$ satisfies (1) is a much simpler application of the definition of a g-inverse: Take any $\gamma^{(0)}$ satisfying (1), whose existence is assumed: then

$\left(\boldsymbol{\Omega}+\boldsymbol{\mu} \boldsymbol{\mu}^{\prime}\right) \gamma=\left(\boldsymbol{\Omega}+\boldsymbol{\mu} \boldsymbol{\mu}^{\prime}\right)\left(\boldsymbol{\Omega}+\boldsymbol{\mu} \boldsymbol{\mu}^{\prime}\right)^{-} \boldsymbol{w}=\left(\boldsymbol{\Omega}+\boldsymbol{\mu} \boldsymbol{\mu}^{\prime}\right)\left(\boldsymbol{\Omega}+\boldsymbol{\mu} \boldsymbol{\mu}^{\prime}\right)^{-}\left(\boldsymbol{\Omega}+\boldsymbol{\mu} \boldsymbol{\mu}^{\prime}\right) \boldsymbol{\gamma}^{(0)}=\left(\boldsymbol{\Omega}+\boldsymbol{\mu} \boldsymbol{\mu}^{\prime}\right) \boldsymbol{\gamma}^{(0)}=\boldsymbol{w} . \quad \mathbf{I}$

Proof of (4): Since $\boldsymbol{\mu}^{\prime} \boldsymbol{\delta}=\boldsymbol{\delta}^{\prime}\left(\boldsymbol{\Omega}+\boldsymbol{\mu} \boldsymbol{\mu}^{\prime}\right) \boldsymbol{\delta}$, it cannot be negative; and if it is zero, then already $\boldsymbol{o}=\left(\boldsymbol{\Omega}+\boldsymbol{\mu} \boldsymbol{\mu}^{\prime}\right) \boldsymbol{\delta}=\boldsymbol{\mu}$, which contradicts (2). $\boldsymbol{\mu}^{\prime} \boldsymbol{\delta} \leq 1$ follows from

$$
\begin{aligned}
\boldsymbol{\mu}^{\prime} \delta & =\delta^{\prime}\left(\Omega+\left(\Omega+\mu \mu^{\prime}\right) \delta \delta^{\prime}\left(\Omega+\mu \mu^{\prime}\right)\right) \delta= \\
& =\delta^{\prime} \boldsymbol{\Omega} \delta+\delta^{\prime}\left(\Omega+\mu \mu^{\prime}\right) \delta \delta^{\prime}\left(\Omega+\mu \mu^{\prime}\right) \delta \geq\left(\mu^{\prime} \delta\right)^{2}
\end{aligned}
$$

(5) is trivial: $\boldsymbol{w}^{\prime} \boldsymbol{\delta}=\boldsymbol{\gamma}^{\prime}\left(\boldsymbol{\Omega}+\boldsymbol{\mu} \boldsymbol{\mu}^{\prime}\right) \boldsymbol{\delta}=\boldsymbol{\gamma}^{\prime} \boldsymbol{\mu}$. I

Statement about "zero" portfolios after (5): From $\left(\boldsymbol{\Omega}+\boldsymbol{\mu} \boldsymbol{\mu}^{\prime}\right) \boldsymbol{x}=\boldsymbol{o}$ follows $\boldsymbol{x}^{\prime}\left(\boldsymbol{\Omega}+\boldsymbol{\mu} \boldsymbol{\mu}^{\prime}\right) \boldsymbol{x}=$ 0. If $\boldsymbol{\mu}^{\prime} \boldsymbol{x}$ were not zero, then $\boldsymbol{x}^{\prime} \boldsymbol{\Omega} \boldsymbol{x}$ would have to be negative, which cannot be since $\boldsymbol{\Omega}$ is nonnegative definite. By the same argument, $\boldsymbol{\Omega} \boldsymbol{x}=\boldsymbol{o}$. Furthermore, $\left(\boldsymbol{\Omega}+\boldsymbol{\mu} \boldsymbol{\mu}^{\prime}\right) \boldsymbol{x}=\boldsymbol{o}$ implies $\boldsymbol{w}^{\prime} \boldsymbol{x}=\boldsymbol{\gamma}^{\prime}\left(\boldsymbol{\Omega}+\boldsymbol{\mu} \boldsymbol{\mu}^{\prime}\right) \boldsymbol{\delta}=0$. I

Proof of Proposition [1]: To show the necessity of the algebraic conditions, we assume they do not hold. First assume (1) is not satisfied, i.e. there is no $\boldsymbol{\gamma}$ with $\boldsymbol{w}=\left(\boldsymbol{\Omega}+\boldsymbol{\mu} \boldsymbol{\mu}^{\prime}\right) \boldsymbol{\gamma}$. Then a vector $\boldsymbol{z}$ exists with $\boldsymbol{z}^{\prime}\left(\boldsymbol{\Omega}+\boldsymbol{\mu} \boldsymbol{\mu}^{\prime}\right)=\boldsymbol{o}^{\prime}$ and $\boldsymbol{z}^{\prime} \boldsymbol{w} \neq 0$. The existence of $\boldsymbol{z}$ contradicts assumption $[i]$ : If $\left(\boldsymbol{\Omega}+\boldsymbol{\mu} \boldsymbol{\mu}^{\prime}\right) \boldsymbol{z}=\boldsymbol{o}$, then $\boldsymbol{\Omega} \boldsymbol{z}=\boldsymbol{o}$ and $\boldsymbol{\mu}^{\prime} \boldsymbol{z}=0$ hold separately, i.e. both $\boldsymbol{z}$ and $-\boldsymbol{z}$ are investment vectors of riskless portfolios with nonnegative expected payoff. Assumption $[i]$ requires in this case that $\boldsymbol{w}^{\prime} \boldsymbol{z} \geq 0$ and $-\boldsymbol{w}^{\prime} \boldsymbol{z} \geq 0$, i.e. $\boldsymbol{w}^{\prime} \boldsymbol{z}=0$.

Now assume (1) holds but (2) is not satisfied, so that

$$
\boldsymbol{\mu}^{\prime} \gamma \leq 0
$$

There are two possibilities. If $\boldsymbol{\mu}=\boldsymbol{o}$, then every portfolio with positive net worth is dominated by the null portfolio, which contradicts assumption $[i i]$. Now assume $\boldsymbol{\mu} \neq \boldsymbol{o}$. This is the only assumption needed to prove proposition [2], which we can therefore use in this proof. We will show that (28) again violates assumption $[i i]$, by constructing, for every portfolio $\boldsymbol{p}$ with positive net worth, another one, whose net worth is $\leq 0$ and which dominates it. If $\mu_{(p)}<0$, then $\boldsymbol{p}$ is dominated by the null portfolio. And due to proposition [2], a $\boldsymbol{p}$ with $\mu_{(p)} \geq 0$ cannot have lower variance than the portfolio with investment vector $\mu_{(p)}\left(1+\pi^{2}\right) \boldsymbol{\delta}$, which has same expected payoff as 
$\boldsymbol{p}$, and whose net worth is, because of (28) together with (5), zero or negative.

To show that conditions (1) and (2) are sufficient, note that (2) implies $\boldsymbol{\mu} \neq \boldsymbol{o}$ and therefore again proposition [2], and that our proof of propositions [4] and [5] given below does not use the assumptions $[i]$ and $[i i]$ directly, but only through conditions (1) and (2). But if propositions [2], [4], and [5] hold, then assumptions [i] and [ii] hold as well. Assumption [i]: If $\boldsymbol{p}$ is risk-free, then proposition [4] says that $\mu_{(p)}=\rho w_{(p)}$ with $\rho>0$; therefore if $\mu_{(p)} \geq 0$ then also $w_{(p)} \geq$ 0 . Assumption $[i i]$ : any absolute minimum variance portfolio with positive expected payoff has positive net worth by proposition [4], and is efficient by proposition [5]. Therefore it is not dominated by anything.

Proof of propositions [2] and [3]: $\pi$ is well defined because of (4). Let $\boldsymbol{t}$ be a portfolio whose investment vector is a scalar multiple of $\boldsymbol{\delta}$. It can be written $\boldsymbol{x}_{(t)}=\mu_{(t)}\left(1+\pi^{2}\right) \boldsymbol{\delta}$, and

$$
\begin{aligned}
\boldsymbol{\Omega}_{(t)} & =\left(\boldsymbol{\Omega}+\boldsymbol{\mu} \boldsymbol{\mu}^{\prime}\right) \boldsymbol{x}_{(t)}-\boldsymbol{\mu} \boldsymbol{\mu}^{\prime} \boldsymbol{x}_{(t)} \\
=\left(\boldsymbol{\Omega}+\boldsymbol{\mu} \boldsymbol{\mu}^{\prime}\right) \mu_{(t)}\left(1+\pi^{2}\right) \boldsymbol{\delta}-\boldsymbol{\mu} \mu_{(t)} & =\boldsymbol{\mu} \mu_{(t)} \pi^{2}
\end{aligned}
$$

Now take any portfolio $\boldsymbol{p}$ and choose $\boldsymbol{t}$ such that $\mu_{(t)}=\mu_{(p)}$. Then the "excess portfolio" $\boldsymbol{e}=\boldsymbol{p}-\boldsymbol{t}$ has zero expected payoff, therefore $\operatorname{cov}(\boldsymbol{t}, \boldsymbol{e})=0$, and

$$
\operatorname{var}(\boldsymbol{p})=\operatorname{var}(\boldsymbol{t})+\operatorname{var}(\boldsymbol{e}) \geq \operatorname{var}(\boldsymbol{t})=\pi^{2} \mu_{(p)}^{2}
$$

This proves inequality (7), and that all portfolios of the form (8) are absolute minimum variance portfolios. In order to show that all absolute minimum variance portfolios have the form (8), assume $\boldsymbol{p}$ satisfies (7) with equality and define $\boldsymbol{t}$ and $\boldsymbol{e}$ as above. Then $\operatorname{cov}(\boldsymbol{t}, \boldsymbol{e})=0$ can be written $\boldsymbol{x}_{(t)} \boldsymbol{\Omega} \boldsymbol{x}_{(p)}=\boldsymbol{x}_{(t)} \boldsymbol{\Omega} \boldsymbol{x}_{(t)}$, therefore $\left(\boldsymbol{x}_{(t)}-\boldsymbol{x}_{(p)}\right)^{\prime} \boldsymbol{\Omega}\left(\boldsymbol{x}_{(t)}-\right.$ $\left.\boldsymbol{x}_{(p)}\right)=0$, therefore $\boldsymbol{\Omega} \boldsymbol{x}_{(e)}=\boldsymbol{o}$, therefore $\left(\boldsymbol{\Omega}+\boldsymbol{\mu} \boldsymbol{\mu}^{\prime}\right) \boldsymbol{x}_{(e)}=\boldsymbol{o}$, i.e. $\boldsymbol{x}_{(e)}$ is the $\boldsymbol{z}_{(p)}$ in $(8)$.

Proof of proposition [4]: (10) follows from (1), which makes the solutions of (3) unique except for "zero" portfolios. $\rho>0$ follows from $(2),(4)$, and (5). 
Proof of proposition [5]: If $\pi=0$, riskless portfolios with positive payoff exist, which, if added to an arbitrary portfolio, will improve its $(\mu, \sigma)$ combination.

Now assume $\pi>0$. Since all portfolios with negative payoff are dominated by the zero portfolio, every absolutely efficient portfolio $\boldsymbol{t}$ satisfies $\mu_{(t)} \geq 0$. And obviously, every absolutely efficient portfolio is an absolute minimum variance portfolio.

Therefore let $\boldsymbol{t}$ be an absolute minimum variance portfolio with $\mu_{(t)} \geq 0$, and take an arbitrary portfolio $\boldsymbol{p}$ with $\mu_{(p)} \geq \mu_{(t)}$ and $\sigma_{(p)} \leq$ $\sigma_{(t)}$. Then

$$
\pi^{2} \mu_{(t)}^{2}=\sigma_{(t)}^{2} \geq \sigma_{(p)}^{2} \geq \pi^{2} \mu_{(p)}^{2} \geq \pi^{2} \mu_{(t)}^{2},
$$

therefore equality holds throughout, i.e. $\sigma_{(t)}=\sigma_{(p)}$ and, since $\pi>0$, also $\mu_{(t)}=\mu_{(p)}$. This shows that $\boldsymbol{t}$ is absolutely efficient. Since both $\boldsymbol{p}$ and $\boldsymbol{t}$ are absolute minimum variance portfolios with equal expected payoff, one can apply proposition [4] to get $w_{(t)}=w_{(p)}$. This proves that $\boldsymbol{t}$ is also efficient.

To show that every risk-free portfolio is efficient, assume $\boldsymbol{t}$ is risk-free, and $\boldsymbol{p}$ satisfies $\mu_{(p)} \geq \mu_{(t)}, \sigma_{(p)} \leq \sigma_{(t)}$, and $w_{(p)} \leq w_{(t)}$. This makes both $\boldsymbol{p}$ and $\boldsymbol{t}$ risk-free, therefore proposition [4] applies: $\mu_{(t)}=\rho w_{(t)}$ and $\mu_{(p)}=\rho w_{(p)}$ for a $\rho>0$. All this together gives $w_{(p)}=w_{(t)}$ and $\mu_{(p)}=\mu_{(t)}$.

For the treatment of minimum variance portfolios we need the following lemma:

Proposition [12]: If $\boldsymbol{\gamma}$ and $\boldsymbol{\delta}$ safisfy (1) and (3), then the matrix $\left[\begin{array}{ll}\boldsymbol{w}^{\prime} \boldsymbol{\gamma} & \boldsymbol{w}^{\prime} \boldsymbol{\delta} \\ \boldsymbol{\mu}^{\prime} \boldsymbol{\gamma} & \boldsymbol{\mu}^{\prime} \boldsymbol{\delta}\end{array}\right]$ is symmetric and nonnegative definite. It is positive definite if and only if $\boldsymbol{w}$ and $\boldsymbol{\mu}$ are linearly independent.

Proof: Symmetry and nonnegative definiteness follow from

$$
\left[\begin{array}{ll}
\boldsymbol{w}^{\prime} \gamma & \boldsymbol{w}^{\prime} \boldsymbol{\delta} \\
\boldsymbol{\mu}^{\prime} \gamma & \boldsymbol{\mu}^{\prime} \boldsymbol{\delta}
\end{array}\right]=\left[\frac{\boldsymbol{w}^{\prime}}{\boldsymbol{\mu}^{\prime}}\right][\gamma \mid \boldsymbol{\delta}]=\left[\frac{\gamma^{\prime}}{\boldsymbol{\delta}^{\prime}}\right]\left(\boldsymbol{\Omega}+\boldsymbol{\mu} \boldsymbol{\mu}^{\prime}\right)[\gamma \mid \boldsymbol{\delta}] .
$$

Now assume

$$
\begin{aligned}
& 0=\left[\begin{array}{ll}
w & \mu
\end{array}\left[\frac{\boldsymbol{\gamma}^{\prime}}{\boldsymbol{\delta}^{\prime}}\right]\left(\boldsymbol{\Omega}+\boldsymbol{\mu} \boldsymbol{\mu}^{\prime}\right)[\boldsymbol{\gamma} \mid \boldsymbol{\delta}]\left[\begin{array}{l}
w \\
\mu
\end{array}\right] .\right. \\
& \text { This implies } \quad \boldsymbol{o}=\left(\boldsymbol{\Omega}+\boldsymbol{\mu} \boldsymbol{\mu}^{\prime}\right)[\boldsymbol{\gamma} \mid \boldsymbol{\delta}]\left[\begin{array}{l}
w \\
\mu
\end{array}\right]=[\boldsymbol{w} \mid \boldsymbol{\mu}]\left[\begin{array}{l}
w \\
\mu
\end{array}\right] .
\end{aligned}
$$


If $\boldsymbol{w}$ and $\boldsymbol{\mu}$ are linearly independent, then for any $w$ and $\mu,(31)$ - and therefore also (30) -implies $w=0$ and $\mu=0$, which means positive definiteness. If $\boldsymbol{w}$ and $\boldsymbol{\mu}$ are linearly dependent, then so are the rows of $\left[\begin{array}{ll}\boldsymbol{w}^{\prime} \gamma & \boldsymbol{w}^{\prime} \boldsymbol{\delta} \\ \boldsymbol{\mu}^{\prime} \gamma & \boldsymbol{\mu}^{\prime} \boldsymbol{\delta}\end{array}\right]$

Proof of propositions [6] and [7]: A portfolio cannot have lower variance than the absolute minimum variance portfolio with equal expected payoff, and if $\mu_{(p)}=\rho w_{(p)}$, then this absolute minimum variance portfolio has also equal net worth. If $\tau=0$, then $\left[\begin{array}{ll}\boldsymbol{w}^{\prime} \boldsymbol{\gamma} & \boldsymbol{w}^{\prime} \boldsymbol{\delta} \\ \boldsymbol{\mu}^{\prime} \boldsymbol{\gamma} & \boldsymbol{\mu}^{\prime} \boldsymbol{\delta}\end{array}\right]$ has zero determinant, therefore $\boldsymbol{w}$ and $\boldsymbol{\mu}$ are collinear by proposition [12], and $\boldsymbol{\mu}=\rho \boldsymbol{w}$ due to proposition [4].

Now assume $\tau>0$. Take any portfolio $\boldsymbol{p}$ and let $\boldsymbol{t}$ be the linear combination of $\boldsymbol{\gamma}$ and $\boldsymbol{\delta}$ which has same net worth and expected payoff as $\boldsymbol{p}$. One verifies that $\boldsymbol{t}$ has investment vector

$$
\boldsymbol{x}_{(t)}=[\boldsymbol{\gamma} \mid \boldsymbol{\delta}]\left[\begin{array}{cc}
\rho^{2} / \tau^{2} & -\rho / \tau^{2} \\
-\rho / \tau^{2} & 1+\pi^{2}+1 / \tau^{2}
\end{array}\right]\left[\begin{array}{l}
w_{(p)} \\
\mu_{(p)}
\end{array}\right] .
$$

With the help of portfolio $\boldsymbol{e}=\boldsymbol{p}-\boldsymbol{t}$ one can check, exactly as in the proof of proposition [2], that inequality (12) holds, and that portfolios of the form (13) are exactly those which minimize standard deviation subject to a given $\mu$ and $w$.

Here is the proof carried out:

$$
\begin{aligned}
\operatorname{var}(\boldsymbol{t})=\boldsymbol{x}_{(t)}^{\prime} \boldsymbol{\Omega x}_{(t)} & =\boldsymbol{x}_{(t)}^{\prime}\left(\boldsymbol{\Omega}+\boldsymbol{\mu} \boldsymbol{\mu}^{\prime}\right) \boldsymbol{x}_{(t)}-\mu_{(p)}^{2} \\
& =\boldsymbol{x}_{(t)}^{\prime}[\boldsymbol{w} \mid \boldsymbol{\mu}]\left[\begin{array}{cc}
\rho^{2} / \tau^{2} & -\rho / \tau^{2} \\
-\rho / \tau^{2} & \pi^{2}+1 / \tau^{2}
\end{array}\right]\left[\begin{array}{c}
w_{(p)} \\
\mu_{(p)}
\end{array}\right] \\
& =\pi^{2} \mu_{(p)}^{2}+\left(\mu_{(p)}-\rho w_{(p)}\right)^{2} / \tau^{2}
\end{aligned}
$$

and the portfolio $\boldsymbol{e}=\boldsymbol{p}-\boldsymbol{t}$ has zero net worth and expected payoff, therefore $\operatorname{cov}(\boldsymbol{t}, \boldsymbol{e})=$ $\boldsymbol{x}_{(t)}^{\prime} \boldsymbol{\Omega} \boldsymbol{x}_{(e)}=\boldsymbol{x}_{(t)}^{\prime}\left(\boldsymbol{\Omega}+\boldsymbol{\mu} \boldsymbol{\mu}^{\prime}\right) \boldsymbol{x}_{(e)}=0$. This gives

$$
\operatorname{var}(\boldsymbol{p})=\operatorname{var}(\boldsymbol{t})+\operatorname{var}(\boldsymbol{e}) \geq \operatorname{var}(\boldsymbol{t}) .
$$

This proves inequality (12), and that all portfolios of the form (13) have minimum variance. In order to show that all minimum variance portfolios have the form (13), assume $\boldsymbol{p}$ satisfies (12) with equality and define $\boldsymbol{t}$ and $\boldsymbol{e}$ as above. Then $\operatorname{cov}(\boldsymbol{t}, \boldsymbol{e})=0$ can be written $\boldsymbol{x}_{(t)}^{\prime} \boldsymbol{\Omega} \boldsymbol{x}_{(p)}=\boldsymbol{x}_{(t)}^{\prime} \boldsymbol{\Omega}_{(t)}$, therefore $\left(\boldsymbol{x}_{(t)}-\boldsymbol{x}_{(p)}\right)^{\prime} \boldsymbol{\Omega}\left(\boldsymbol{x}_{(t)}-\boldsymbol{x}_{(p)}\right)=0$, therefore $\boldsymbol{\Omega} \boldsymbol{x}_{(e)}=\boldsymbol{o}$, therefore $\left(\boldsymbol{\Omega}+\boldsymbol{\mu} \boldsymbol{\mu}^{\prime}\right) \boldsymbol{x}_{(e)}=\boldsymbol{o}$, i.e. $\boldsymbol{x}_{(e)}$ is the $\boldsymbol{z}_{(p)}$ in (13). I

Proof of the two possibilities for the "feasible set" as laid out in the text after propostion [7]: Every portfolio is a minimum variance portfolio, if for every portfolio $\boldsymbol{p}$, the 
"excess portfolio" $\boldsymbol{e}$, as defined in the proof of proposition [6], has zero variance. if this is not the case, then at least one portfolio exists with zero expected payoff and net worth, but nonzero variance. Such a portfolio has zero covariance with any minimum variance portfolio, and by adding appropriate multiples of this portfolio to any minimum variance portfolio one obtains portfolios with arbitrarily higher standard deviation. In other words, from every point on the minimum variance set, the "feasible set" extends indefinitely eastward, i.e. in direction of increasing $\sigma$.

Proof of proposition [8]: Any portfolio $\boldsymbol{p}$ for which (16) does not hold is dominated by the absolute minimum variance portfolio with expected return $\mu_{(p)}$.

Call this absolute minimum variance portfolio $\boldsymbol{t}: \mu_{(t)}=\mu_{(p)}, w_{(t)}=\frac{\mu_{(p)}}{\rho}<w_{(p)}$ because (16) does not hold, and $\sigma_{(t)}<\sigma_{(p)}$ because $\boldsymbol{p}$ cannot be an absolute minimum variance portfolio itself.

For nonnegative $w_{(p)}$ we therefore still have to show: if the minimum variance portfolio $\boldsymbol{p}$ with nonnegative net worth satisfies (16), then it is efficient. If it satisfies (16) with equality, it is an absolute minimum variance portfolio, and therefore efficient by proposition [5]. Now assume $\boldsymbol{p}$ satisfies (16) as a strict inequality, which is only possible if $\tau>0$. Therefore $\boldsymbol{p}$ satisfies (12) with equality. Furthermore assume portfolio $\boldsymbol{q}$ has expected payoff $\mu_{(q)} \geq \mu_{(p)}$, variance $\sigma_{(q)}^{2} \leq \sigma_{(p)}^{2}$, and net worth $w_{(q)} \leq w_{(p)}$. Then we have to show that none of these three inequalities is strict. Since $\rho>0$, one can concatenate the inequalities:

$$
\rho w_{(q)} \leq \rho w_{(p)}<\mu_{(p)} \leq \mu_{(q)} .
$$

The second and third item can therefore not be farther apart than the first and fourth:

$$
\left(\mu_{(p)}-\rho w_{(p)}\right)^{2} \leq\left(\mu_{(q)}-\rho w_{(q)}\right)^{2} .
$$

And since $0 \leq \mu_{(p)}$ due to $w_{(p)} \geq 0$ and (16), it follows from $\mu_{(p)} \leq \mu_{(q)}$ that

$$
\mu_{(p)}^{2} \leq \mu_{(q)}^{2}
$$

Divide (33) by $\tau^{2}$, multiply (34) by $\pi^{2}$, and add the two inequalities:

$$
\pi^{2} \mu_{(p)}^{2}+\frac{1}{\tau^{2}}\left(\mu_{(p)}-\rho w_{(p)}\right)^{2} \leq \pi^{2} \mu_{(q)}^{2}+\frac{1}{\tau^{2}}\left(\mu_{(q)}-\rho w_{(q)}\right)^{2} .
$$

By assumption, the left side is $\sigma_{(p)}^{2}$, and by (12), the right side is $\leq \sigma_{(q)}^{2}$. (35) implies therefore $\sigma_{(p)}^{2} \leq \sigma_{(q)}^{2}$, which together with the 
assumption that $\sigma_{(q)}^{2} \leq \sigma_{(p)}^{2}$ gives $\sigma_{(p)}^{2}=\sigma_{(q)}^{2}$. This means, equality holds in (35), therefore equality holds in both inequalities which were added together to get (35). But if equality holds in (33), (32) becomes

$$
\rho w_{(q)}=\rho w_{(p)}<\mu_{(p)}=\mu_{(q)} .
$$

The argument for negative $w$ is exactly parallel, one merely has to interchange the roles of (16) and (17), of $\boldsymbol{w}$ and $\boldsymbol{\mu}$, and consequently use the "global" minimum variance portfolio $\boldsymbol{g}$ instead of the "absolute" minimum variance portfolio $\boldsymbol{t}$. I

Here is this parallel proof: If $\mu_{(p)} \leq 0$, then (16) and (17) hold if and only if (17) holds. Any portfolio $\boldsymbol{p}$ for which (17) does not hold is dominated by the global minimum variance portfolio with net worth $w_{(p)}$. Call this global minimum variance portfolio $\boldsymbol{g}$ : $w_{(g)}=w_{(p)}, \mu_{(g)}=\frac{\rho}{1+\pi^{2} \tau^{2}} w_{(p)}>\mu_{(p)}$ because (17) does not hold, and $\sigma_{(g)}^{2}<\sigma_{(p)}^{2}$, since $\boldsymbol{p}$ cannot be a global minimum variance portfolio itself.

Finally we have to show: if the minimum variance portfolio $\boldsymbol{p}$ with negative net worth satisfies (17), then it is efficient. Again we must distinguish two cases. If $\boldsymbol{p}$ satisfies (17) with equality, then it is, due to (14), a global minimum variance portfolio among all portfolios with same net worth $w_{(p)}$. If $\pi=0$, it is furthermore risk-free and therefore efficient by proposition [5]. Therefore assume $\pi \neq 0$, and take an arbitrary portfolio $\boldsymbol{q}$ with $w_{(q)} \leq w_{(p)}$ (therefore $\left.w_{(q)}^{2} \geq w_{(p)}^{2}\right), \mu_{(q)} \geq \mu_{(p)}$, and $\sigma_{(q)} \leq \sigma_{(p)}$. Then

$$
\frac{\pi^{2} \rho^{2}}{1+\pi^{2} \rho^{2}} w_{(p)}^{2}=\sigma_{(p)}^{2} \geq \sigma_{(q)}^{2} \geq \frac{\pi^{2} \rho^{2}}{1+\pi^{2} \rho^{2}} w_{(q)}^{2} \geq \frac{\pi^{2} \rho^{2}}{1+\pi^{2} \rho^{2}} w_{(p)}^{2},
$$

therefore equality holds throughout, i.e. $\sigma_{(p)}=\sigma_{(q)}$ and, since $\pi>0$, also $w_{(p)}=w_{(q)}$. From (14) follows therefore $\left(\mu_{(q)}-\frac{\rho}{1+\pi^{2} \tau^{2}} w_{(q)}\right)^{2}=0$, therefore

$$
\mu_{(q)}=\frac{\rho}{1+\pi^{2} \tau^{2}} w_{(q)}=\frac{\rho}{1+\pi^{2} \tau^{2}} w_{(p)}=\mu_{(p)}
$$

Now assume $\boldsymbol{p}$ satisfies (17) as a strict inequality, and portfolio $\boldsymbol{q}$ has expected payoff $\mu_{(q)} \geq \mu_{(p)}$, variance $\sigma_{(q)}^{2} \leq \sigma_{(p)}^{2}$, and net worth $w_{(q)} \leq w_{(p)}$. Again we want to show that none of these three inequalities is strict. Since $\rho>0$, one can concatenate the inequalities:

$$
\frac{\rho}{1+\pi^{2} \tau^{2}} w_{(q)} \leq \frac{\rho}{1+\pi^{2} \tau^{2}} w_{(p)}<\mu_{(p)} \leq \mu_{(q)} .
$$

The second and third item can therefore not be farther apart than the first and fourth:

$$
\left(\mu_{(p)}-\frac{\rho}{1+\pi^{2} \tau^{2}} w_{(p)}\right)^{2} \leq\left(\mu_{(q)}-\frac{\rho}{1+\pi^{2} \tau^{2}} w_{(q)}\right)^{2} .
$$

And since $w_{(p)} \leq 0$, it follows from $w_{(p)} \geq w_{(q)}$ that

$$
w_{(p)}^{2} \leq w_{(q)}^{2}
$$

Again, the strict inequality (17) contradicts (12) unless $\tau^{2}>0$, therfore we can multiply (iii) by $\frac{1+\pi^{2} \tau^{2}}{\tau^{2}}$, multiply (34) by $\frac{\pi^{2} \rho^{2}}{1+\pi^{2} \tau^{2}}$, and add the two inequalities:

$\frac{\pi^{2} \rho^{2}}{1+\pi^{2} \tau^{2}} w_{(p)}^{2}+\frac{1+\pi^{2} \tau^{2}}{\tau^{2}}\left(\mu_{(p)}-\frac{\rho}{1+\pi^{2} \tau^{2}} w_{(p)}\right)^{2} \leq \frac{\pi^{2} \rho^{2}}{1+\pi^{2} \tau^{2}} w_{(q)}^{2}+\frac{1+\pi^{2} \tau^{2}}{\tau^{2}}\left(\mu_{(q)}-\frac{\rho}{1+\pi^{2} \tau^{2}} w_{(q)}\right)^{2}$. 
By (14), the left side is $\sigma_{(p)}^{2}$, and the right side is the variance of the minimum variance portfolio with same net worth and expected return as $\boldsymbol{q}$, therefore it is $\leq \sigma_{(q)}^{2}$. $(v)$ implies therefore $\sigma_{(p)}^{2} \leq \sigma_{(q)}^{2}$, hence $\sigma_{(p)}^{2}=\sigma_{(q)}^{2}$. This means, equality holds in $(v)$, therefore equality holds in both inequalities which were added together to get $(v)$, therefore equality holds in (iii). Therefore (ii) becomes

$$
\frac{\rho}{1+\pi^{2} \tau^{2}} w_{(q)}=\frac{\rho}{1+\pi^{2} \tau^{2}} w_{(p)}<\mu_{(p)}=\mu_{(q)}
$$

In other words, variance, expected payoff, and net worth of $\boldsymbol{q}$ are equal to those of $\boldsymbol{p}$. I

Proof of proposition [9]: By solving (12) for $w_{(p)}$ one sees that the net worth of a minimum variance portfolio with expected payoff $\mu$ and variance $\sigma^{2}$ satisfies (19) with a " \pm " instead of the minus sign in front of the square root. This formula remains valid for $\tau=0$. If the net worth with the plus sign is indeed stricly larger than that with the minus sign, it cannot belong to an efficient portfolio, since it is dominated by that with the minus sign. If therefore an efficient portfolio exists with the given $\mu, \sigma$ combination, it must be the minimum variance portfolio with the minus sign.

The portfolios in the range delineated above clearly satisfy (16), and those with $\mu \geq 0$ therefore also (17). If $\mu<0$ then (18) implies $\frac{\sigma^{2}}{1+\pi^{2} \tau^{2}} \geq \pi^{2} \mu^{2}$, therefore $\sqrt{\sigma^{2}-\pi^{2} \mu^{2}} \geq$ $\pi^{4} \tau^{2} \mu$, which gives (17). I

Proof of proposition [10]: If $\tau>0$, then it follows from (13), premultiplied by $\boldsymbol{\Omega}+\boldsymbol{\mu} \boldsymbol{\mu}^{\prime}$, that every minimum variance portfolio $\boldsymbol{p}$ satisfies

$$
\boldsymbol{\Omega x}_{(p)}=[\boldsymbol{w} \mid \boldsymbol{\mu}]\left[\begin{array}{cc}
\rho^{2} / \tau^{2} & -\rho / \tau^{2} \\
-\rho / \tau^{2} & \pi^{2}+1 / \tau^{2}
\end{array}\right]\left[\begin{array}{l}
w_{(p)} \\
\mu_{(p)}
\end{array}\right]
$$

Therefore

$$
\begin{aligned}
\operatorname{cov}(\boldsymbol{q}, \boldsymbol{p}) & =\boldsymbol{x}_{(q)}^{\prime} \boldsymbol{\Omega} \boldsymbol{x}_{(p)} \\
& =\pi^{2} \mu_{(q)} \mu_{(p)}+\frac{1}{\tau^{2}}\left(\mu_{(q)}-\rho w_{(q)}\right)\left(\mu_{(p)}-\rho w_{(p)}\right) \\
& \geq w_{(p)} w_{(q)} \pi^{2} \rho^{2} .
\end{aligned}
$$

For this last inequality, (16) was applied four times. If $\tau=0$, (20) holds with equality, due to (29).

Proof of proposition [11]: First assume $\tau^{2}>0$. If $\boldsymbol{p}$ is efficient and not "global" with $w_{(p)}<0$, it cannot be global at all, therefore $\left(1+\pi^{2} \tau^{2}\right) \mu_{(p)}-\rho w_{(p)}>0$ and $\mu_{(p)}-\rho w_{(p)} \geq 0$, i.e. $\theta_{(p)}$ is well defined and nonnegative. The assertion follows since (37) can be rewritten

$$
\operatorname{cov}(\boldsymbol{q}, \boldsymbol{p})=\left(\mu_{(q)}-\theta_{(p)} w_{(q)}\right) \frac{\left(1+\pi^{2} \tau^{2}\right) \mu_{(p)}-\rho w_{(p)}}{\tau^{2}} .
$$


Here is a step between (37) and this "rewritten" version:

$$
\tau^{2} \operatorname{cov}(\boldsymbol{q}, \boldsymbol{p})=\mu_{(q)}\left(\left(1+\pi^{2} \tau^{2}\right) \mu_{(p)}-\rho w_{(p)}\right)-\rho w_{(q)}\left(\mu_{(p)}-\rho w_{(p)}\right)
$$

Next assume $\tau^{2}>0$ and $\boldsymbol{p}$ is global minimum variance, i.e. $\mu_{(p)}=$ $\frac{\rho}{1+\pi^{2} \tau^{2}} w_{(p)}$. This together with (36) gives $\boldsymbol{\Omega}_{\boldsymbol{x}_{(p)}}=\frac{\pi^{2} \rho^{2}}{1+\pi^{2} \tau^{2}} w_{(p)} \boldsymbol{w}$, and if furthermore $w_{(p)}<0$, the special case criterion follows.

If $\tau^{2}=0$, then $\mu_{(q)}=\rho w_{(q)}, \mu_{(p)}=\rho w_{(p)}$, and $\boldsymbol{p}$ is an absolute minimum variance portfolio. Therefore by $(29) \operatorname{cov}(\boldsymbol{q}, \boldsymbol{p})=$ $\rho \pi^{2} \mu_{(q)} w_{(p)}$. If $w_{(p)}>0$, the criterion with $\theta_{(p)}$ (which, by definition, is zero in this case) holds. If $w_{(p)}<0$, we are in the special case, because $\boldsymbol{p}$ is also a global minimum variance portfolio, and the criterion holds because $\operatorname{cov}(\boldsymbol{q}, \boldsymbol{p})=\rho^{2} \pi^{2} w_{(q)} w_{(p)}$. The case $w_{(p)}=0$ is ruled out by $\sigma_{(p)}>0$.

Proof of Geometric Interpretation of $\theta_{(p)}$ : Totally differentiating (12) as an equality with respect to $\sigma$ and $\mu$ gives

$$
\tau^{2} \sigma_{(p)} d \sigma_{(p)}-\pi^{2} \tau^{2} \mu_{(p)} d \mu_{(p)}=\left(\mu_{(p)}-\rho w_{(p)}\right) d \mu_{(p)} .
$$

The slope of the tangent is therefore

$$
\frac{\partial \mu_{(p)}}{\partial \sigma_{(p)}}=\frac{\tau^{2} \sigma_{(p)}}{\left(\pi^{2} \tau^{2}+1\right) \mu_{(p)}-\rho w_{(p)}},
$$

and the height of the intersection of the tangent with the $\mu$-axis is

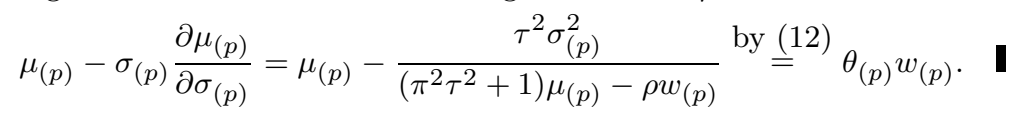

Proof of the zero beta market line equation $(i)$ : If $\boldsymbol{p}$ is a minimum variance portfolio which does not have minimum variance among all portfolios with same net worth $w_{(p)}$, then $\tau^{2}>0$ and $\left(1+\pi^{2} \tau^{2}\right) \mu_{(p)}-\rho w_{(p)} \neq 0$. (36) can be written as

$$
\boldsymbol{\Omega} \boldsymbol{x}_{(p)}=\frac{1}{\tau^{2}}\left(\left(1+\pi^{2} \tau^{2}\right) \mu_{(p)}-\rho w_{(p)}\right)\left(\boldsymbol{\mu}-\theta_{(p)} \boldsymbol{w}\right)
$$

Premultiply $(v i)$ once by $\boldsymbol{x}_{(q)}^{\prime}$ and once by $\boldsymbol{x}_{(p)}^{\prime}$, to get

$$
\begin{aligned}
& \boldsymbol{x}_{(q)}^{\prime} \boldsymbol{\Omega} \boldsymbol{x}_{(p)}=\left(\mu_{(q)}-\theta_{(p)} w_{(q)}\right) \frac{1}{\tau^{2}}\left(\left(1+\pi^{2} \tau^{2}\right) \mu_{(p)}-\rho w_{(p)}\right) \\
& \boldsymbol{x}_{(p)}^{\prime} \boldsymbol{\Omega} \boldsymbol{x}_{(p)}=\left(\mu_{(p)}-\theta_{(p)} w_{(p)}\right) \frac{1}{\tau^{2}}\left(\left(1+\pi^{2} \tau^{2}\right) \mu_{(p)}-\rho w_{(p)}\right)
\end{aligned}
$$

Since the assumptions imply that $\operatorname{var}(\boldsymbol{p}) \neq 0$, we can divide to get

$$
\beta_{(q . p)}=\frac{\mu_{(q)}-\theta_{(p)} w_{(q)}}{\mu_{(p)}-\theta_{(p)} w_{(p)}}, \quad \text { therefore }(i) .
$$


Proof of the market line equation (23): The condition that $\boldsymbol{p}$ is a minimum variance portfolio but not an absolute minimum variance portfolio presupposes $\tau^{2}>0$. Define $\boldsymbol{e}$ to be the "excess" portfolio with $\boldsymbol{x}_{(e)}=\boldsymbol{x}_{(p)}-\mu_{(p)}(1+\pi)^{2} \boldsymbol{\delta}$, which already played a role in the proof of proposition [2]; then

$$
\boldsymbol{\Omega}_{(e)}=\boldsymbol{\Omega} \boldsymbol{x}_{(p)}-\pi^{2} \mu_{(p)} \boldsymbol{\mu}=\frac{1}{\tau^{2}}(\boldsymbol{\mu}-\rho \boldsymbol{w})\left(\mu_{(p)}-\rho w_{(p)}\right) .
$$

Premultiply (38) once by $\boldsymbol{x}_{(q)}^{\prime}$ and once by $\boldsymbol{x}_{(p)}^{\prime}$ to get

$$
\begin{aligned}
& \operatorname{cov}(\boldsymbol{q}, \boldsymbol{e})=\operatorname{cov}(\boldsymbol{q}, \boldsymbol{p})-\pi^{2} \mu_{(q)} \mu_{(p)}=\frac{1}{\tau^{2}}\left(\mu_{(q)}-\rho w_{(q)}\right)\left(\mu_{(p)}-\rho w_{(p)}\right) \\
& \operatorname{cov}(\boldsymbol{p}, \boldsymbol{e})=\operatorname{var}(\boldsymbol{p})-\pi^{2} \mu_{(p)}^{2}=\frac{1}{\tau^{2}}\left(\mu_{(p)}-\rho w_{(p)}\right)^{2}
\end{aligned}
$$

Since the assumptions imply that $\operatorname{var}(\boldsymbol{p})>\pi^{2} \mu_{(p)}^{2}$, we can divide to get

$$
\beta_{(q . p)}^{(r)}=\frac{\mu_{(q)}-\rho w_{(q)}}{\mu_{(p)}-\rho w_{(p)}}, \quad \text { therefore }(23) .
$$

Proof that (24) is the most similar minimum variance portfolio: For every pair of scalars $\alpha$ and $\gamma$, we need to show $\mathcal{E}\left[\left(\boldsymbol{q}-\boldsymbol{q}^{*}-\alpha \boldsymbol{u}-\right.\right.$ $\left.\gamma \boldsymbol{p})^{2}\right] \geq 0$. This is true if the cross product terms $\mathcal{E}\left[\left(\boldsymbol{q}-\boldsymbol{q}^{*}\right) \boldsymbol{u}\right]=0$ and $\mathcal{E}\left[\left(\boldsymbol{q}-\boldsymbol{q}^{*}\right) \boldsymbol{p}\right]=0$ or, since $\mathcal{E}\left[\boldsymbol{q}-\boldsymbol{q}^{*}\right]=0$, it suffices that $\operatorname{cov}\left(\boldsymbol{q}-\boldsymbol{q}^{*}, \boldsymbol{u}\right)=$ 0 and $\operatorname{cov}\left(\boldsymbol{q}-\boldsymbol{q}^{*}, \boldsymbol{p}\right)=0$. This can be written

$$
\begin{aligned}
& \operatorname{cov}\left(\boldsymbol{q}-\mu_{(q)} \boldsymbol{u}, \boldsymbol{u}\right)=\beta_{(q \cdot p)}^{(r)} \operatorname{cov}\left(\boldsymbol{p}-\mu_{(p)} \boldsymbol{u}, \boldsymbol{u}\right) \\
& \operatorname{cov}\left(\boldsymbol{q}-\mu_{(q)} \boldsymbol{u}, \boldsymbol{p}\right)=\beta_{(q \cdot p)}^{(r)} \operatorname{cov}\left(\boldsymbol{p}-\mu_{(p)} \boldsymbol{u}, \boldsymbol{p}\right) .
\end{aligned}
$$

Using (29) one sees that the first equality holds with both sides zero, and that the second can be reduced to the definition of $\beta_{(q \cdot p)}^{(r)}$. I

Equation (25) follows from

$$
\operatorname{cov}(\boldsymbol{p}, \boldsymbol{e})=\operatorname{cov}(\boldsymbol{q}, \boldsymbol{e})+\operatorname{cov}(\boldsymbol{r}, \boldsymbol{e}) .
$$

Proof that the set described in Section 7 is indeed the investor's choice set: First consider the case $\bar{w} \leq 0$. Since there are no absolutely efficient portfolios with negative net worth, the set described above consists of all efficient portfolios with net worth $\bar{w}$ (i.e. it is equal to the choice set of an investor with equality wealth constraint). We have to show 
that the $(\mu, \sigma)$ combination of every portfolio $\boldsymbol{q}$ with $w_{(q)}<\bar{w}$ can be improved by an efficient portfolio with net worth $\bar{w}$. If $\mu_{(q)}<\frac{\rho}{1+\pi^{2} \tau^{2}} \bar{w}$, then the global minimum variance portfolio $\boldsymbol{g}$ with net worth $\bar{w}$ has expected payoff $\mu_{(g)}=\frac{\rho}{1+\pi^{2} \tau^{2}} \bar{w}>\mu_{(q)}$ and variance $\sigma_{(g)}^{2}=\pi^{2} \rho^{2} \bar{w}^{2} /\left(1+\pi^{2} \rho^{2}\right)<\pi^{2} \rho^{2} w_{(q)}^{2} /\left(1+\pi^{2} \rho^{2}\right) \leq \sigma_{(q)}^{2}$. If $\mu_{(q)} \geq \frac{\rho}{1+\pi^{2} \tau^{2}} \bar{w}$, then necessarily $\tau>0$, and we will show that $\boldsymbol{q}$ has higher variance than the minimum variance portfolio $\boldsymbol{p}$ with $w_{(p)}=\bar{w}$ and $\mu_{(p)}=\mu_{(q)}$. From $\mu_{(q)} \geq \frac{\rho}{1+\pi^{2} \tau^{2}} \bar{w}>\frac{\rho}{1+\pi^{2} \tau^{2}} w_{(q)}$ follows $\left(\mu_{(q)}-\frac{\rho}{1+\pi^{2} \tau^{2}} \bar{w}\right)^{2}<\left(\mu_{(q)}-\frac{\rho}{1+\pi^{2} \tau^{2}} w_{(q)}\right)^{2}$, and from $0 \geq \bar{w}>w_{(q)}$ follows $\bar{w}^{2}<w_{(q)}^{2}$, therefore by (14)

$$
\begin{aligned}
\sigma_{(p)}^{2} & =\frac{\pi^{2} \rho^{2}}{1+\pi^{2} \tau^{2}} \bar{w}^{2}+\frac{1+\pi^{2} \tau^{2}}{\tau^{2}}\left(\mu_{(q)}-\frac{\rho}{1+\pi^{2} \tau^{2}} \bar{w}\right)^{2}< \\
& <\frac{\pi^{2} \rho^{2}}{1+\pi^{2} \tau^{2}} w_{(q)}^{2}+\frac{1+\pi^{2} \tau^{2}}{\tau^{2}}\left(\mu_{(q)}-\frac{\rho}{1+\pi^{2} \tau^{2}} w_{(q)}\right)^{2} \leq \sigma_{(q)}^{2} .
\end{aligned}
$$

Now look at the case $\bar{w}>0$. We will show that every portfolio $\boldsymbol{p}$ with $w_{(p)} \leq \bar{w}$, which does not lie in the choice set, is "dominated" (with respect to $\mu$ and $\sigma$, though not necessarily $w$ ) by a portfolio in this choice set. Here we have to distinguish whether $\mu_{(p)}<0,0 \leq \mu_{(p)}<\rho \bar{w}$, or $\mu_{(p)} \geq \rho \bar{w}$. First case: If $\mu_{(p)}<0$, then $\boldsymbol{p}$ is "dominated" by the null portfolio. If $\pi>0$, the null portfolio lies in the choice set, and if $\pi=0$, then the null portfolio itself is "dominated" by the riskless portfolio with net worth $\bar{w}$, which lies in the choice set. Second case, $0 \leq \mu_{(p)}<\rho \bar{w}$ : If $\pi=0$, then $\boldsymbol{p}$ is again "dominated" by the riskless portfolio with net worth $\bar{w}$. If $\pi>0$, it is "dominated" by the absolute minimum variance portfolio with same expected payoff as $\boldsymbol{p}$, whose net worth is $\mu_{(p)} / \rho<\bar{w}$. Third case (which can only happen if $\tau>0$ ): If $\mu_{(p)} \geq \rho \bar{w}$, then $\mu_{(p)} \geq \rho \bar{w} \geq \rho w_{(p)}$ gives $\left(\mu_{(p)}-\rho w_{(p)}\right)^{2} \geq\left(\mu_{(p)}-\rho \bar{w}\right)^{2}$. Hence by $(12), \sigma_{(p)}^{2} \geq \pi^{2} \mu_{(p)}^{2}+\frac{1}{\tau^{2}}\left(\mu_{(p)}-\rho w_{(p)}\right)^{2} \geq$ $\pi^{2} \mu_{(p)}^{2}+\frac{1}{\tau^{2}}\left(\mu_{(p)}-\rho \bar{w}\right)^{2}$. The right side is the variance of a minimum variance portfolio with expected payoff $\mu_{(p)}$ and net worth $\bar{w}$. Therefore $\boldsymbol{p}$ is dominated by this efficient portfolio, unless it is an efficient portfolio with $w_{(p)}=\bar{w}$ itself. I

\section{References}

[BuSerA1.987ajper. Mean-variance portfolio selection with either a singular or nonsingular variance-covariance matrix. Journal of Financial and Quantitative Analysis, 12:347-361, Sept. 1977.

[EhFtar(1996);bar. Mean-variance efficiency when investors are not required to invest all their money. Journal of Economic Theory, 50(1):214-18, Feb. 1990.

[Faria(Haño),] Foundations of Finance. Basic Books, New York, 1976.

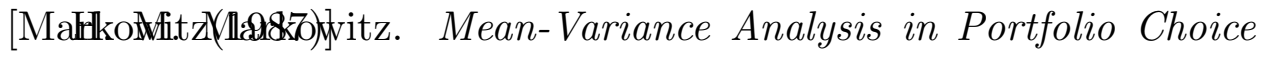
and Capital Markets. Basil Blackwell, New York, 1987.

[MeRtonf1972) iton. An analytic derivation of the efficient portfolio frontier. Journal of Financial and Quantitative Analysis, 7:18511872, 1972. 
[RaQ(.1873)ajo. Linear Statistical Inference and Its Applications. Wiley, New York, second edition, 1973.

[RoR(18T0 D).] A critique of the asset pricing theory's tests, part I: On past and potential testability of the theory. Journal of Financial Economics, 4:129-176, 1977.

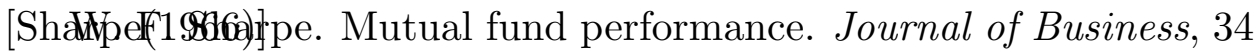
(1/II):119-138, 1966. 